\title{
Good mutations available in all sizes
}

Beneficial mutations are the essential ingredients of adaptive evolution, but how do the numbers of slightly beneficial and substantially beneficial mutations compare? New research on the evolution of bacterial resistance to an antibiotic shows that the assumption that most beneficial mutations have small effects is not always true.

In an environment to which the wild type of a species is well adapted, the few possible mutations that increase fitness are likely to be exponentially distributed. In other words, the number of mutations that increase fitness by a small amount will be greater than the number that increase it by a larger amount.
This is because the beneficial mutations constitute the tail of a fitness distribution, in which the wild type lies close to the right side. However, if the wild type is less fit, as can happen when the environment changes suddenly, the beneficial mutations will constitute a larger section of the overall distribution, which could be a range of different shapes, many of which would not be exponential.

To test under what circumstances distributions are not exponential, the authors isolated mutants of the $r p o B$ gene in Pseudomonas aeruginosa that conferred resistance to high levels of rifampicin. They then assessed the fitness (by measuring growth rate) of the mutants at a range of rifampicin concentrations. At low concentrations, the wild type had high fitness, so there were fewer beneficial mutants and those that there were followed an exponential distribution. However, at higher concentrations of rifampicin, the wild type was unfit compared with the mutants, so there were more beneficial mutations, and, crucially, these no longer fitted an exponential model.

\section{The authors} sought mechanistic explanations for the fitness distributions by looking at how the positions of the mutations in the $r p o B$ sequence related to the interactions between RpoB and rifampicin. A large number of amino acids on RNA polymerase are involved in direct interactions with rifampicin, and mutations at these positions cause a large increase in resistance and fitness. By contrast, a small number of amino acids are involved in indirect interactions between rifampicin and RNA polymerase, and mutations at these positions give a small increase in resistance and fitness. The distribution of the mutations is therefore not exponential; for an exponential distribution, most mutations would have small beneficial effects.

For a full understanding of adaptive evolution, the precise shapes of the distribution of fitness effects will need to be worked out, both in this system and in a range of non-microbial situations. This work shows that in many cases the shape will not be exponential, especially when organisms are adapting to new environments.

Patrick Goymer

ORIGINAL RESEARCH PAPER Maclean, R. C. \& Buckling, A. The distribution of fitness effects of beneficial mutations in Pseudomonas aeruginosa. PLoS Genet. 5, e1000406 (2009) 